\title{
Gambogic acid increases the sensitivity to paclitaxel in drug-resistant triple-negative breast cancer via the SHH signaling pathway
}

\author{
YONGHUI WANG $^{1}$, YANA SUI ${ }^{2}$ and YINGGANG TAO ${ }^{3}$ \\ ${ }^{1}$ Department of Breast Surgery, Weifang People's Hospital; ${ }^{2}$ Emergency Department of Weifang Traditional Chinese Hospital; \\ ${ }^{3}$ Department of General Surgery, Weifang Second People's Hospital, Weifang, Shandong 261041, P.R. China
}

Received August 20, 2017; Accepted September 6, 2019

DOI: $10.3892 / \mathrm{mmr} .2019 .10697$

\begin{abstract}
Paclitaxel is the most frequently used therapy regimen for triple-negative breast cancer (TNBC). However, chemoresistance frequently occurs, leading to enhanced failure rates of chemotherapy in TNBC; therefore, novel biological therapies are urgently needed. Gambogic acid (GA) has potent anticancer effects and inhibits tumor growth in several types of human cancer. However, the effects of GA on paclitaxel-resistant TNBC remain unknown. In the present study, the Cell Counting Kit-8 assay was used to examine the effect of GA and/or paclitaxel on the viability of TNBC cells; flow cytometry was used to examine the effects of GA on cell apoptosis; and western blotting and reverse transcription-quantitative PCR were used to determine the effects of GA on the expression of sonic hedgehog ( $\mathrm{SHH})$ signaling pathway target genes. The present results indicated that GA significantly inhibited the viability and enhanced the rate of apoptosis in paclitaxel-resistant MDA-MB-231 cells via activating the SHH signaling pathway. In vivo experiments confirmed that GA treatment enhanced the sensitivity of MDA-MB-231 cells to paclitaxel via the SHH signaling pathway. In conclusion, the combination of GA with paclitaxel may increase the antitumor effects on paclitaxel-resistant TNBC via downregulating the SHH signaling pathway.
\end{abstract}

\section{Introduction}

Breast cancer is the second leading cause of cancer-related mortality in women in the United States, with annual 255,180 diagnosed cases and 41,070 deaths reported in 2017 (1). Triple-negative breast cancer (TNBC), which accounts for $\sim 15 \%$

Correspondence to: Dr Yinggang Tao, Department of General Surgery, Weifang Second People's Hospital, 7 Yuanxiao Street, Kuiwen Qu, Weifang, Shandong 261041, P.R. China

E-mail: tearthnut@163.com

Key words: gambogic acid, sonic hedgehog signaling pathway, triple-negative breast cancer, paclitaxel, chemoresistance of all breast cancers, represents a collection of cancers that do not express estrogen receptor (ER), progesterone receptor (PR) and erb-b2 receptor tyrosine kinase 2 (HER2) (2). Given the lack of expression of ER, PR and HER2, the effective treatment options for patients with TNBC are typically limited to cytotoxic therapies. However, the effect of chemotherapy is often diminished by the development of drug resistance (2).

Acquired drug resistance refers to the scenario wherein patients that are initially sensitive to chemotherapy eventually develop resistance during treatment $(2,3)$. Exploring how cancer cells can eliminate the damaging effect of chemotherapeutic drugs and how to improve drug sensitivity are important strategies for treating cancer. Therefore, novel chemotherapy strategies must be developed. Combining chemotherapeutics with traditional Chinese medicine can result in cooperative effects, reduction in the required doses of chemotherapeutics, and consequently low drug toxicity, decreased side effects and reduced drug resistance $(4,5)$.

Gambogic acid (GA), which is the main active ingredient of gamboge, is a brownish to orange dry resin that is secreted from Garcinia hanburyi, a plant widely found in nature. Previous studies (6-8) have revealed that GA has potent antitumor effects on TNBC cells in vitro and in vivo. In addition, GA could reverse docetaxel resistance in gastric cancer, cisplatin resistance in lung cancer, doxorubicin resistance in breast and ovarian cancers, 5-fluorouracil resistance in colorectal cancer, and multidrug resistance in epithelial cancer (9-12). However, the effect of GA on paclitaxel-resistant TNBC remains unknown, and the mechanisms by which GA induces anticancer effects remain to be elucidated.

In the present study, the results revealed that GA treatment inhibited proliferation and induced apoptosis of paclitaxel-resistant TNBC cells through the sonic hedgehog (SHH) signaling pathway. The current study reported that GA overcame drug resistance and may therefore serve as a combination treatment for TNBC therapy.

\section{Materials and methods}

Cell culture and establishment of paclitaxel-resistant cell lines. The human TNBC cell lines MDA-MB-231 and MDA-MB-468 were obtained from The Cell Bank of Type 
Culture Collection of the Chinese Academy of Sciences (Shanghai, China) and cultured in DMEM medium (Gibco; Thermo Fisher Scientific, Inc.) supplemented with 10\% FBS (HyClone; GE Healthcare Life Sciences) in a humidified incubator at $37^{\circ} \mathrm{C}$ with $5 \% \mathrm{CO}_{2}$. To establish paclitaxel-resistant cell lines, MDA-MB-231 and MDA-MB-468 cells were cultured with $1 \mu \mathrm{M}$ paclitaxel (Sigma-Aldrich; Merck $\mathrm{KGaA}$ ) for 60 days. The medium with $1 \mu \mathrm{M}$ paclitaxel was changed every 3 days. GA (Key Laboratory of Carcinogenesis and Intervention, China Pharmaceutical University, Nanjing, China) was dissolved in DMSO (Sigma-Aldrich; Merck $\mathrm{KGaA}$ ) and stored at $-20^{\circ} \mathrm{C}$.

Cell proliferation assay via real-time cell impedance analysis (RTCA). For RTCA, the xCELLigence system (Roche Applied Science) was applied to dynamically monitor cell proliferation rates. The assay was executed according to the manufacturer's instructions. The impedance was indicated as cell index. RTCA software, supplied by the manufacturer, was used to analyze the measurements.

Cell viability assay. Cell viability was examined by the Cell Counting Kit-8 (CCK8) assay (Beyotime Institute of Biotechnology). Briefly, MDA-MB-231 and MDA-MB-468 cells were trypsinized and seeded at 3,000 cells/well in a 96-well plate. After culturing for the indicated time $(0,24,48$ and $72 \mathrm{~h}), 10 \mu \mathrm{l}$ of CCK- 8 reagent was added into each well and incubated at $37^{\circ} \mathrm{C}$. After $3 \mathrm{~h}$, the absorbance of each well was measured using a Multiskan MK3 spectrophotometer set at a wavelength of $450 \mathrm{~nm}$.

Colony formation assay. Paclitaxel-resistant MDA-MB-231 and MDA-MB-468 cells (400 cells/well) were seeded in 6 -well plates. After 1 week of culture, the colonies were fixed with methanol at room temperature for $20 \mathrm{~min}$, stained with $0.1 \%$ crystal violet (Beyotime Institute of Biotechnology) for $20 \mathrm{~min}$, and the images of the stained colonies were captured using a CKX41 light microscope (Olympus Corporation). The number of colonies was counted from the images.

Flow cytometry. The apoptotic rate of cells was examined via the Annexin $\mathrm{V}$ and propidium iodide (PI) double-staining method, according to the manufacturer's instructions (Nanjing KeyGen Biotech Co., Ltd.). The stained cells were immediately analyzed via flow cytometry using ModFit LT 3.0 (Verity Software House, Inc.).

Reverse transcription-quantitative PCR (RT-qPCR). Total RNA from cells was isolated using TRIzol reagent (Invitrogen; Thermo Fisher Scientific, Inc.), according to the manufacturer's protocol. cDNA was synthesized from $1 \mu \mathrm{g}$ total RNA using a PrimeScript RT Reagent kit with gDNA Eraser (Takara Biotechnology Co., Ltd.), according to the manufacturer's instructions. qPCR was performed using SYBR Premix Ex Taq (Takara Biotechnology Co., Ltd.). The thermocycling conditions were: $5 \mathrm{~min}$ at $95^{\circ} \mathrm{C}$, followed by 40 cycles of $30 \mathrm{sec}$ at $95^{\circ} \mathrm{C}, 60 \mathrm{sec}$ at $60^{\circ} \mathrm{C}$ and $30 \mathrm{sec}$ at $72^{\circ} \mathrm{C} ; 1 \mathrm{sec}$ at $99^{\circ} \mathrm{C}$; $15 \mathrm{sec}$ at $59^{\circ} \mathrm{C} ; 1 \mathrm{sec}$ at $95^{\circ} \mathrm{C}$; followed by cooling to $40^{\circ} \mathrm{C}$. $\beta$-actin was used as the internal reference control. Relative fold changes in mRNA expression were calculated using the formula $2^{-\Delta \Delta \mathrm{Cq}}(13)$. The primer sequences were: $\mathrm{SHH}$, forward 5'-CCCAATTACAACCCCGACATC-3' and reverse 5'-TCA CCCGCAGTTTCACTCCT-3'; patched 1 (PTCH1), forward 5'-TGAGACTGACCACGGCCTG-3' and reverse 5'-ACCCTC AGTTGGAGCTGCTTC-3'; GLI family zinc finger 1 (GLI1), forward 5'-AGGGCTGCAGTAAAGCCTTCA-3' and reverse 5'-CTTGACATGTTTTCGCAGCG-3'; and $\beta$-actin, forward 5'-GATCATTGCTCCTCCTGAGC-3' and reverse 5'-ACT CCTGCTTGCTGATCCAC-3'.

Western blot analysis. The protein expression levels of $\mathrm{SHH}$, PTCH1, GLI1, Bcl-2, BAX, and cleaved caspase-3 were analyzed via western blot assay. Total proteins were extracted from tissues using the T-PER Tissue Protein Extraction Reagent (Pierce; Thermo Fisher Scientific, Inc.). Protein concentrations were determined using a bicinchoninic acid (BCA) Protein Assay Kit (Pierce; Thermo Fisher Scientific, Inc.). Proteins $(20 \mu \mathrm{g})$ were separated by SDS-PAGE $(10 \%$ gels) and transferred to polyvinylidene fluoride membranes (EMD Millipore). Membranes were blocked at room temperature with $5 \%$ non-fat milk for $1 \mathrm{~h}$ and incubated at $4^{\circ} \mathrm{C}$ overnight with the following antibodies: $\mathrm{SHH}(1: 200$; cat. no. ab19897; Abcam), PTCH1 (1:200; cat. no. ab53715; Abcam), GLI1 (1:200; cat. no. ab49314; Abcam), Bcl-2 (1:500; cat. no. ab196495; Abcam), cleaved caspase-3 (1:500; cat. no. ab2302; Abcam), BAX (1:500; cat. no. ab53154; Abcam) and $\beta$-actin (1:500; cat. no. ab8227; Abcam), then treated with a Horseradish peroxidase-labeled goat anti-rabbit secondary antibody (1:1,000; cat. no. ab150077; Abcam) for $2 \mathrm{~h}$ at room temperature. The protein bands were visualized using an enhanced chemiluminescence system (Beyotime Institute of Biotechnology). $\beta$-actin served as a loading control for normalization.

Immunohistochemistry. The expression of SHH in the xenograft tumors was detected as described previously (4), using an anti-SHH antibody (1:200; cat. no. ab19897; Abcam). The tumors were rinsed in PBS, followed by fixation with $3 \%$ neutral formalin for $24 \mathrm{~h}$ at room temperature. Paraffin-embedded sections of tumor tissue ( $4 \mu \mathrm{m}$ thick) were deparaffinized in xylene, rehydrated via graded alcohol solutions, blocked in methanol containing $3 \%$ hydrogen peroxide for $10 \mathrm{~min}$ at room temperature, and then incubated with $\mathrm{SHH}$ antibody at $4^{\circ} \mathrm{C}$ overnight. Following rinsing with PBS solution, biotinylated goat anti-rabbit serum $\operatorname{IgG}$ (1:2,000; cat. no. ab64256; Abcam) was used as secondary antibody for $2 \mathrm{~h}$ at room temperature and streptavidin peroxidase complex reagent was applied for $1 \mathrm{~h}$ at room temperature. Finally, the sections were incubated in a 3,3'-diaminobenzidine solution at room temperature for $10 \mathrm{~min}$ and then counterstained with hematoxylin for $3 \mathrm{~min}$ at room temperature. Ten randomly selected visual fields per section were examined under a light microscope in order to evaluate the $\mathrm{SHH}$ expression.

Animal studies. All experiments involving animals were approved by the Animal Care and Welfare Committee of WeiFang People's Hospital (permit no. WF2016032702). Female BALB/c nude mice $(n=24$; age, 4 weeks; weight, 20-25 g) were procured from the Laboratory Animal Center of YangZhou University (YangZhou, China) and maintained 

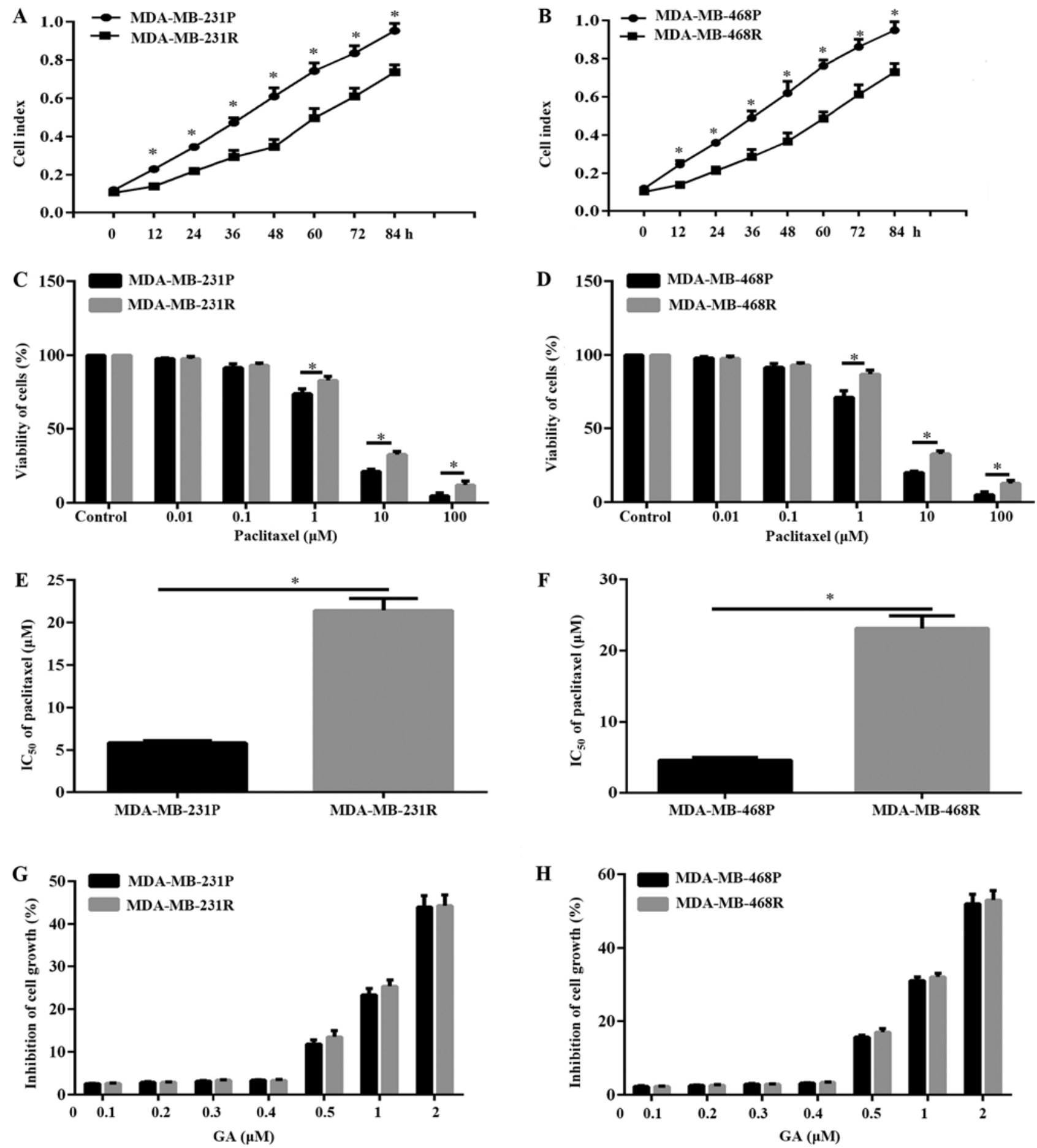

Figure 1. Establishment of the paclitaxel-resistant TNBC cells. (A) Comparison of growth rate in parental cells and paclitaxel-resistant cells in MDA-MB-231 and (B) MDA-MB-468 lines. (C) Paclitaxel sensitivity assay in MDA-MB-231 and (D) MDA-MB-468 cells. Cell viability was measured by CCK- 8 assay and presented as a percentage of viable cells in the paclitaxel-treated group relative to the untreated control. (E) $\mathrm{IC}_{50}$ values of paclitaxel at $24 \mathrm{~h}$ for MDA-MB-231 and (F) MDA-MB-468 cells. (G) MDA-MB-231 and (H) MDA-MB-468 cells were treated with the indicated concentrations of GA for $8 \mathrm{~h}$. Cell viability was detected by the CCK- 8 assay and presented as a percentage of viable cells in the GA-treated group relative to the untreated control. "P<0.05. TNBC, triple-negative breast cancer; CCK-8, Cell Counting Kit-8; GA, gambogic acid; P, parental; R, resistant.

under specific pathogen-free conditions $\left(26-28^{\circ} \mathrm{C}\right.$, air pressure difference 10-20 kPa, 10-h light/14-h dark cycle, food and water provided ad libitum). Animal health and behavior were monitored every day.

Paclitaxel-resistant MDA-MB-231 $\left(5 \times 10^{6}\right.$ cells/mouse, suspended in $200 \mu 1$ normal saline) were subcutaneously injected into the right flanks of athymic nude mice. Seven days post-injection, the mice were divided into four groups ( $\mathrm{n}=6$ per group) and subjected to different treatments as follows: Group 1, saline control administration; group 2, $5 \mathrm{mg} / \mathrm{kg}$ paclitaxel administration; group 3,2 $\mathrm{mg} / \mathrm{kg} \mathrm{GA}$ administration; group 4, $2 \mathrm{mg} / \mathrm{kg} \mathrm{GA}+5 \mathrm{mg} / \mathrm{kg}$ paclitaxel combined administration. The intravenous administrations were done once every other day for 14 days. Tumor volume $\left(\mathrm{mm}^{3}\right)$ was 
A
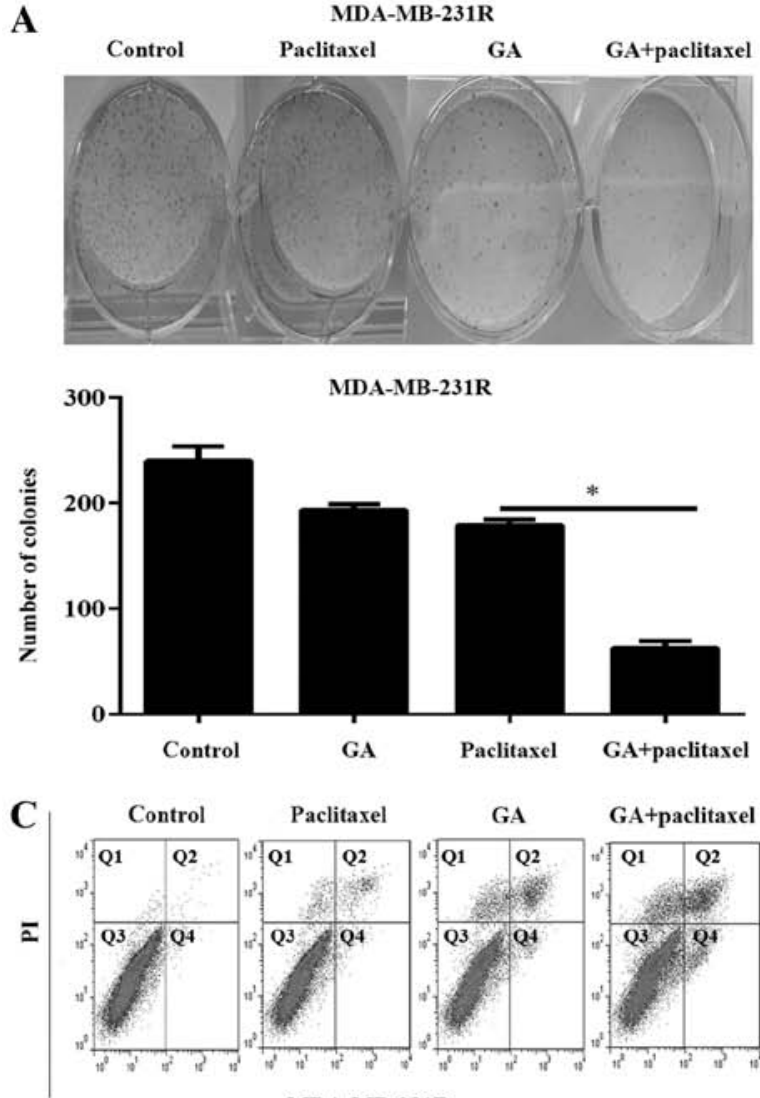

MDA-MB-231R

Annexin $V$

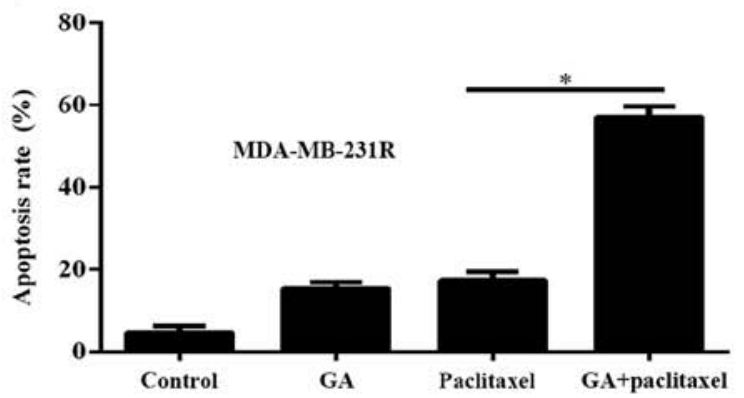

E

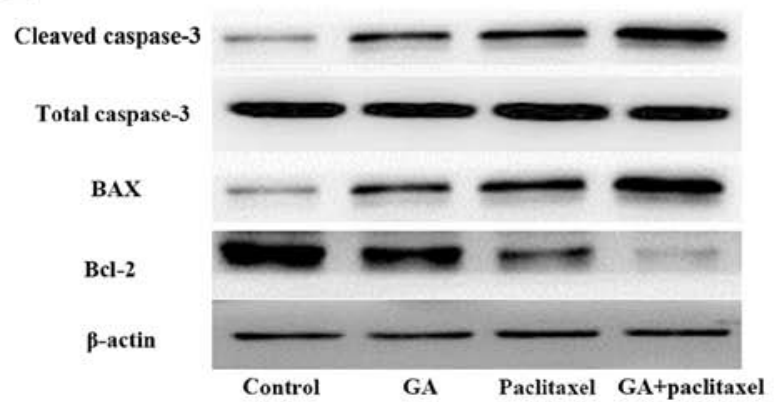

B
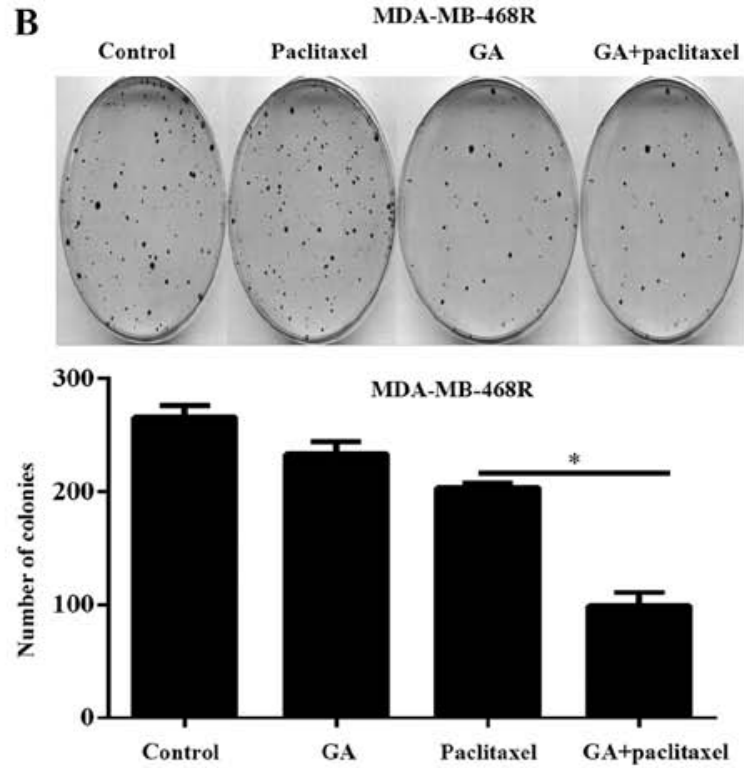

D

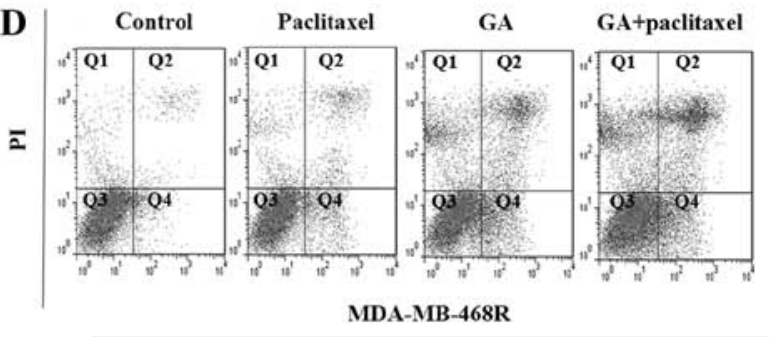

Annexin V

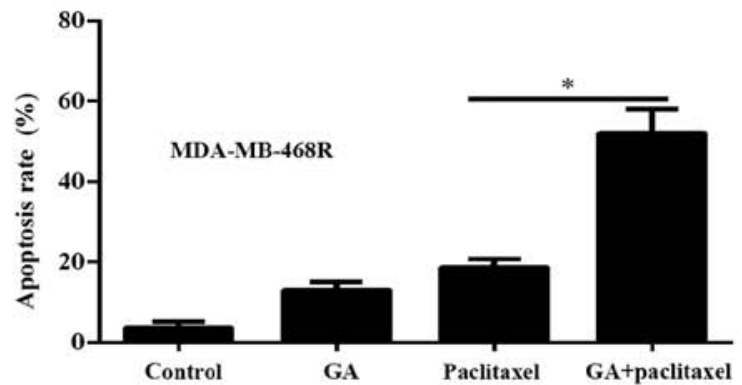

$\mathbf{F}$

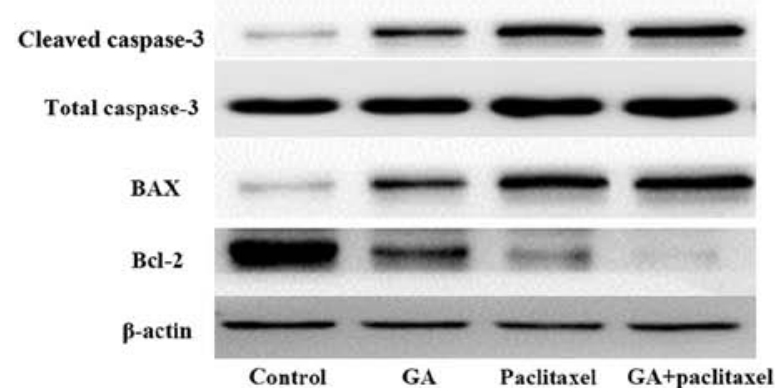

Figure 2. Combination of paclitaxel and GA inhibits colony formation and induces apoptosis. (A) Representative images from colony formations assays of MDA-MB-231R and (B) MDA-MB-468R cells treated with paclitaxel and/or GA. (C) Representative plots and quantification from flow cytometry analysis of apoptosis for MDA-MB-231R and (D) MDA-MB-468R cells. The sum of Q2 + Q4 were counted as apoptotic cells. (E) Western blot analysis of cleaved caspase-3, BAX and Bcl-2 expression in MDA-MB-231R and (F) MDA-MB-468R cells. " $\mathrm{P}<0.05$. GA, gambogic acid; R, resistant; PI, propidium iodide.

calculated every 3 days using the formula $V=0.5 x$ length $x$ width $^{2}$. All mice were sacrificed by cervical dislocation after 14 days of treatments. Tumors were collected and photographed at 2 weeks after treatments.
Statistical analysis. All experiments were performed in triplicate. Unless otherwise indicated, the data were presented as mean $\pm \mathrm{SD}$. Statistical significance was determined using SPSS 13.0 (SPSS, Inc.). Differences between two groups 
A

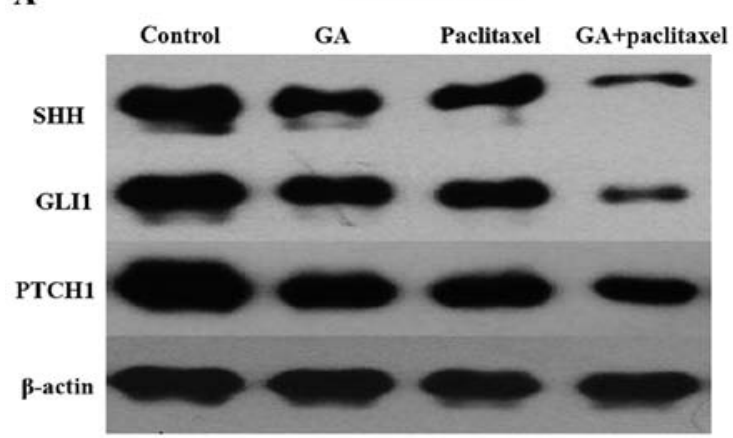

C

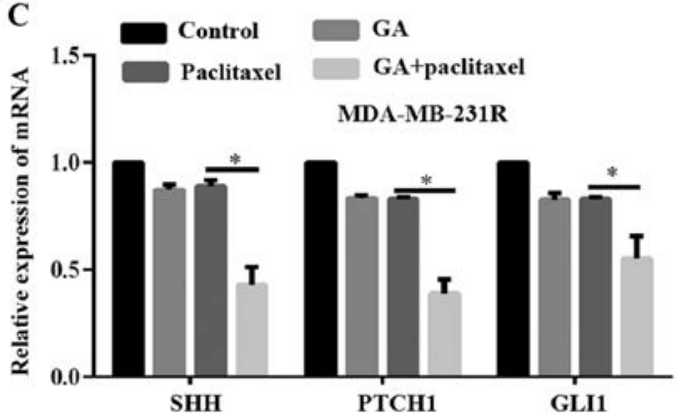

B
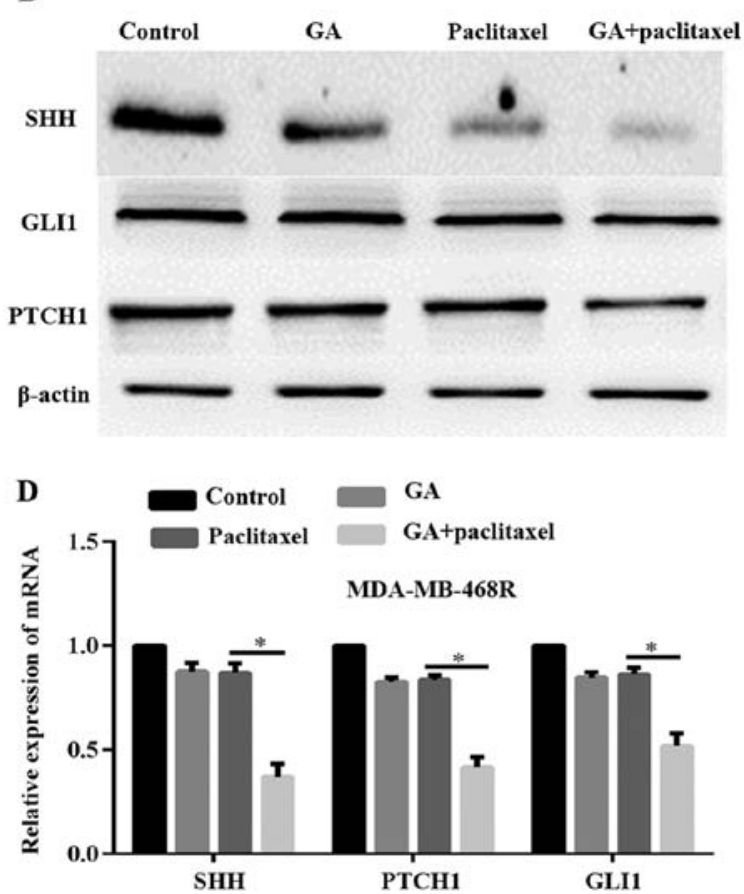

Figure 3. Combination of GA and paclitaxel inhibits the SHH signaling pathway. MDA-MB-231R and MDA-MB-468R cells were treated with GA or paclitaxel alone, or with the combination, for $24 \mathrm{~h}$. (A and B) Western blot and (C and D) qPCR analysis results of the expression levels SHH, and the target genes GLI1 and PTCH1. "P<0.05. GA, gambogic acid; SHH, sonic hedgehog; R, resistant; GLI1, GLI family zinc finger 1; PTCH1, patched 1.

were assessed using Student's t-test (two-tailed). Data from more than two groups were analyzed using one-way ANOVA followed by Tukey's test. $\mathrm{P}<0.05$ was considered to indicate a statistically significant difference.

\section{Results}

Establishment of the paclitaxel-resistant TNBC cells. To explore the potential anticancer effect of GA on paclitaxel-resistant TNBC, the TNBC cell lines MDA-MB-231 and MDA-MB-468 were cultured with $1 \mu \mathrm{M}$ paclitaxel for 60 days in order to establish paclitaxel-resistant cells (termed thereafter MDA-MB-231R and MDA-MB-468R, respectively). MDA-MB-231R and MDA-MB-468R cells grew significantly slower compared with the drug-sensitive parental cells (termed MDA-MB-231P and MDA-MB-468P, respectively; Fig. $1 \mathrm{~A}$ and $\mathrm{B})$. In order to confirm the establishment of paclitaxel-resistant TNBC cells, the sensitivity to paclitaxel of MDA-MB-231 cells and MDA-MB-468 cells was analyzed. Results of CCK- 8 assay demonstrated that the sensitivity to paclitaxel of MDA-MB-231R and MDA-MB-468R cells was significantly reduced compared with the MDA-MB-231P and MDA-MB-468P cells, respectively (Fig. $1 \mathrm{C}$ and $\mathrm{D}$ ). In addition, $\mathrm{The} \mathrm{IC}_{50}$ of paclitaxel in MDA-MB-231R and MDA-MB-468R cells increased by $\sim 4$-fold and $\sim 5$-fold, respectively, compared with their parental cells (Fig. 1E and F). The dose of $5 \mu \mathrm{M}$ paclitaxel was selected for further experiments, which was approximately the $\mathrm{IC}_{50}$ of paclitaxel in the parental MDA-MB-231P and MDA-MB-468P cells.

To investigate the potential cytotoxic and antiproliferation effects of GA, MDA-MB-231 and MDA-MB-468, parental and resistant, cells were cultured with different concentrations of GA for $8 \mathrm{~h}$. The results illustrated that treatment with GA at concentrations $>0.4 \mu \mathrm{M}$ induced significant inhibition of MDA-MB-231 and MDA-MB-468 cell numbers, in both the parental and resistant cell lines, in a dose-dependent manner (Fig. $1 \mathrm{G}$ and $\mathrm{H}$ ). To avoid the inhibitory effects of GA, the non-inhibitory GA concentration of $0.2 \mu \mathrm{M}$ was selected for subsequent experiments in the present study.

Combination of paclitaxel and $G A$ inhibits proliferation and induces apoptosis. MDA-MB-231R and MDA-MB-468R cells were treated with $5 \mu \mathrm{M}$ paclitaxel and/or $0.2 \mu \mathrm{M}$ GA in the subsequent experiments. To investigate the anticancer effect of GA and paclitaxel on MDA-MB-231R and MDA-MB-468R cells, colony formation assays were performed (Fig. 2A and B). Treatment of MDA-MB-231R and MDA-MB-468R cells with paclitaxel or GA alone only weakly inhibited cell colony formation. However, the combination treatment resulted in fewer colonies compared with paclitaxel or GA treatment alone (Fig. 2A and B). In addition, the cell apoptosis rate was determined by flow cytometry analysis. The combination of paclitaxel and GA resulted in increased apoptosis compared with paclitaxel or GA treatment alone (Fig. 3C and D). Furthermore, western blot analysis was used to detect the expression of cleaved caspase-3, Bcl-2 and BAX in the MDA-MB-231R and MDA-MB-468R cells following the indicated treatments. The results demonstrated that the combination treatment significantly increased the expression of cleaved caspase- 3 and BAX and decreased the expression of Bcl-2 than either agent alone (Fig. 2E and F). These results demonstrated that GA enhanced the cytotoxicity effects of paclitaxel in resistant TNBC cells. 
GA sensitizes $M D A-M B-231 R$ and $M D A-M B-468 R$ cells to paclitaxel by inhibiting the $S H H$ pathway in vitro. A previous study has reported that the $\mathrm{SHH}$ signaling pathway is associated with paclitaxel resistance in breast cancer (14). To investigate the underlying mechanisms of GA reducing MDA-MB-231R and MDA-MB-468R cell drug resistance, the expression levels of SHH and its target genes GLI1 and PTCH1 were determined both at the protein and mRNA level, via western blot and qPCR analyses, respectively. The results demonstrated that treatment of MDA-MB-231R and MDA-MB-468R cells with paclitaxel or GA alone only weakly decreased the expression of SHH, GLI1 and PTCH1, both at the protein and mRNA level (Fig. 3A-D). However, the combination of paclitaxel and GA significantly inhibited the expression of SHH, GLI1 and PTCH1 compared to either agent alone (Fig. 3A-D). These data indicated that combination of GA with paclitaxel enhanced the antitumor effects of paclitaxel in resistant TNBC cells through inactivation of the SHH signaling pathway in vitro.

GA increases the sensitivity to paclitaxel in resistant TNBC cells in vivo. Next, the effect of GA on the sensitivity toward paclitaxel of MDA-MB-231R cells was investigated in vivo, via a mouse model. After 14 days of treatments, the tumors were removed and photographed (Fig. 4A). The volume and weight measurements of the excised xenograft tumors revealed that combination of GA with paclitaxel resulted in significantly reduced tumor growth (Fig. 4B and C). These data indicated that GA significantly enhanced the antitumor effect of paclitaxel in paclitaxel-resistant TNBC cells.

GA sensitizes TNBC to paclitaxel through inhibiting the SHH pathway in vivo. To explore the potential mechanism of GA enhancing drug sensitivity in vivo, immunohistochemistry and qPCR analyses were performed on the xenograft tumor tissues. The results revealed that paclitaxel or GA treatment alone only weakly inhibited the expression of $\mathrm{SHH}$ in the tumor tissues (Fig. 5A and B). However, the combination paclitaxel and GA treatment significantly decreased the expression of SHH in the xenograft tumors compared with paclitaxel alone (Fig. 5A and B), suggesting that GA sensitized TNBC cells to paclitaxel via inhibiting the SHH pathway in vivo.

To further investigate the mechanism of the combination treatment on tumor growth, the protein expression levels of cleaved caspase-3, Bcl-2 and BAX were detected by western blotting. In accordance with the results of the in vitro experiments, the combination of paclitaxel and GA markedly enhanced the expression levels of cleaved caspase-3 and BAX and decreased the expression levels of Bcl-2 in the xenograft tumors, compared with either agent alone (Fig. 5C).

\section{Discussion}

Drug resistance is a serious problem that leads to therapeutic failure in breast cancer. The mechanisms underlying drug resistance are poorly understood and overcoming drug resistance is an important endeavor that must be achieved in order to increase the overall survival of patients with cancer. Natural plant products, such as GA, have been extensively investigated for their potential to reverse drug resistance, which would be
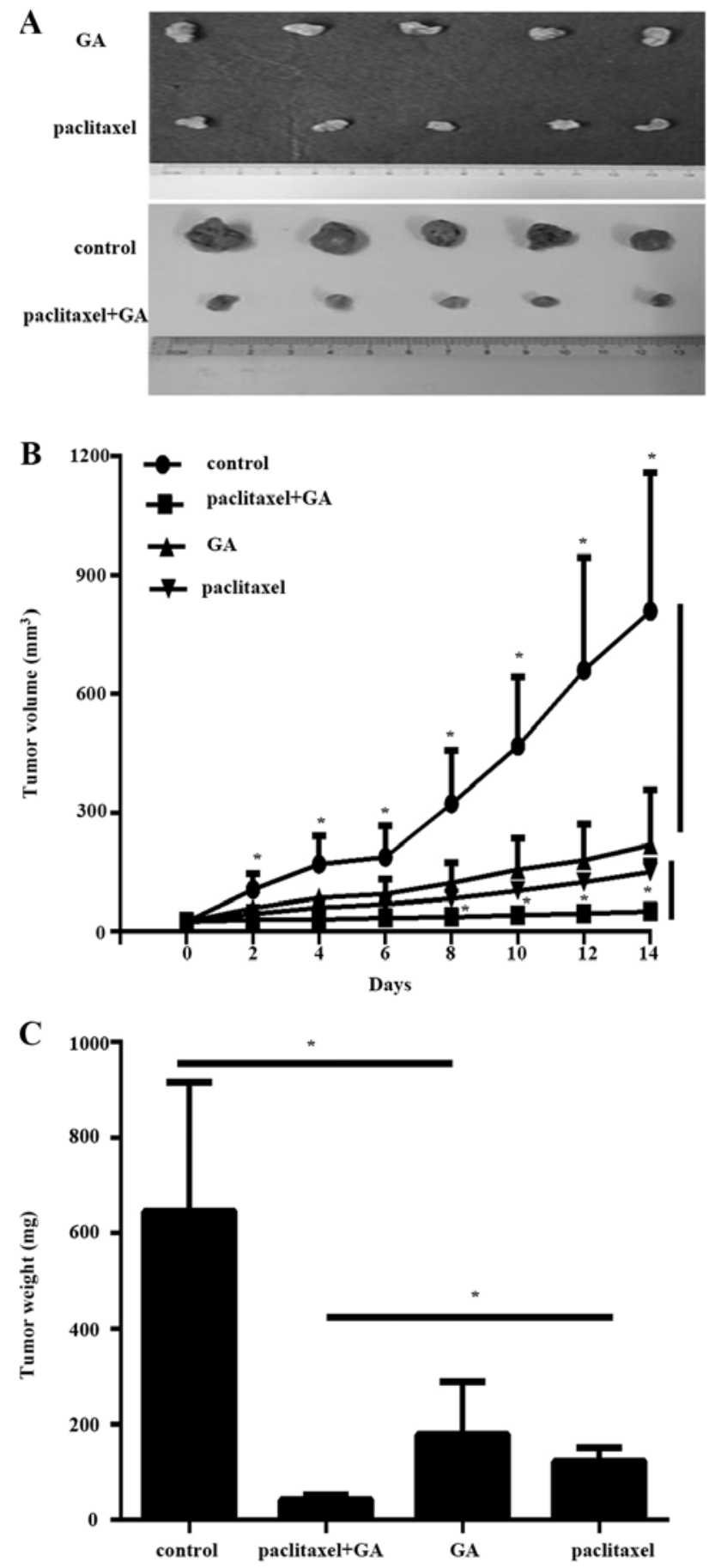

Figure 4. Combination of GA with paclitaxel inhibits resistant TNBC growth in vivo. Female nude mice were injected with MDA-MB-231R cells for seven days, then treated with GA and/or paclitaxel for 14 days ( $n=6$ mice per group). (A) Photographs of the xenografts tumors. (B) Tumor volumes were calculated throughout the experiment duration. (C) Tumor weights were measured at the end of the experiment. Data are presented as mean \pm SD $(n=6$ mice per group). ${ }^{*} \mathrm{P}<0.05$ with comparisons shown by lines. GA, gambogic acid; TNBC, triple-negative breast cancer; R, resistant.

beneficial in the success of chemotherapy treatments. In the present study, the mechanism by which GA overcomes drug resistance was investigated in TNBC.

GA is a candidate drug that has been approved by the China Food and Drug Administration for a phase II clinical trial in solid tumor therapy (15). Previous studies showed that GA could induce apoptosis in a broad range of human 
A

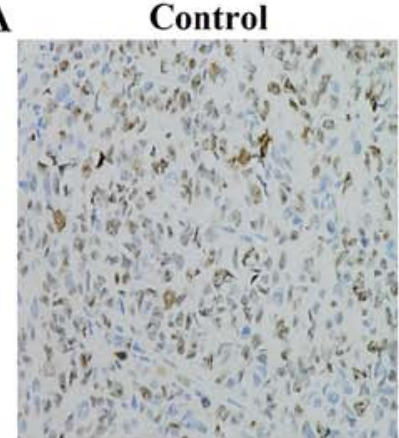

Paclitaxel

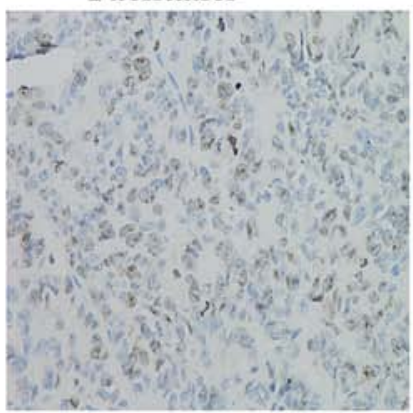

Paclitaxel + GA

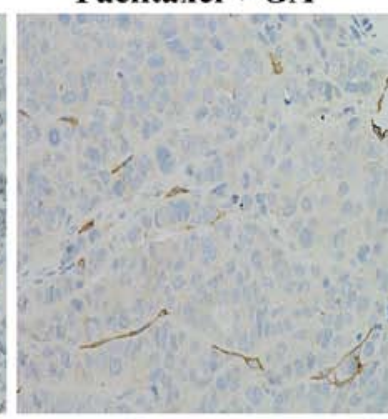

GA

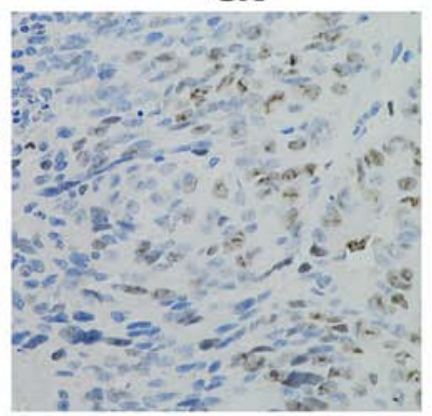

B

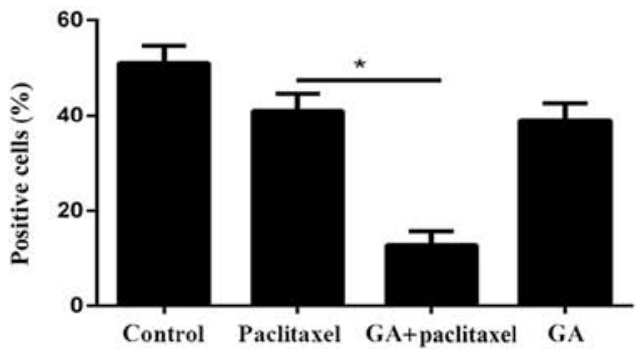

C

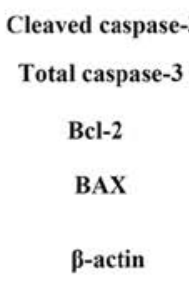

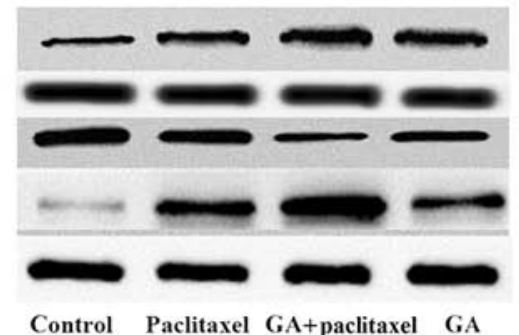

Control Paclitaxel GA+paclitaxel GA

Figure 5. GA sensitizes TNBC to paclitaxel via inhibiting the SHH pathway in vivo. (A) Representative images form immunohistochemical staining of SHH protein in the xenograft tumors derived from MDA-MB-231R cells and treated with paclitaxel and/or GA (magnification, x200). (B) Quantification of the percentage of SHH-positive cells in the tumor sections. (C) Western blot analysis of the expression levels of apoptosis-related proteins in the xenograft tumors. ${ }^{*} \mathrm{P}<0.05$ with comparisons shown by lines. GA, gambogic acid; TNBC, triple-negative breast cancer; SHH, sonic hedgehog; R, resistant.

cancers $(4,6,7,16,17)$. Furthermore, GA treatment combined with chemotherapeutic drugs resulted in a synergistic effect on the chemotherapeutic efficacy against drug-resistant cancer cells (9-12). Therefore, GA could possibly overcome the paclitaxel resistance in TNBC by promoting apoptosis.

To explore the potential of GA to overcome paclitaxel resistance in TNBC cells, the present study first established the paclitaxel-resistant TNBC cancer cell lines MDA-MB-231R and MDA-MB-468R. Compared with paclitaxel alone, the combined application of paclitaxel and GA synergistically reduced the colony formation abilities of MDA-MB-231R and MDA-MB-468R cells. The results of flow cytometry and western blot analyses demonstrated that the additive effect of GA to paclitaxel was accompanied by an enhanced apoptosis. Similarly, other previous studies have also reported that GA combined with other chemotherapeutic drugs could enhance the apoptosis rate of drugs in a broad range of cancer cells (9-12). In summary, the present study demonstrated that GA significantly decreased the cell viability and enhanced the cells apoptosis of MDA-MB-231R and MDA-MB-468R cells induced by paclitaxel. These data indicated that GA could increase the sensitivity to paclitaxel in paclitaxel-resistant TNBC.

The SHH signaling pathway is crucial for regulating various cell processes, such as proliferation, cell growth, survival, inflammatory response and apoptosis (18). Enhanced activation of the SHH pathway is linked to the development and progression of several types of cancer and to chemotherapy resistance (18). A recent study reported that the inactivation of the SHH signaling pathway is involved in the success of chemotherapy-induced apoptosis in breast cancer (14). In the present study, the results indicated that GA might enhance the drug sensitivity of paclitaxel in human breast cancer by inactivating the SHH signaling pathway.
Apoptosis constitutes a fundamental intrinsic mechanism underlying tumor suppression, and the resistance of apoptosis is a well-established aspect of cancer (19). The activation of the $\mathrm{SHH}$ pathway promotes cell survival, upregulates Bcl-2 and downregulates cleaved caspase- 3 and BAX, which are key regulators of apoptosis (14). In the present study, the results demonstrated that GA and paclitaxel treatment significantly increased the expression of cleaved caspase- 3 and BAX and decreased the expression of bcl-2, via modulating the activation of the SHH signaling pathway. These findings suggested that GA reversed drug resistance in TNBC cells by inhibiting the SHH signaling pathway, indicating that GA could be a potentially useful natural therapy for overcoming drug resistance in vitro. A limitation of the present study is that only the SHH pathway was investigated in regards to the role of GA in paclitaxel-resistant TNBC. Future studies will investigate the potential involvement of the other signaling pathways in GA paclitaxel sensitization.

In the present study, a xenograft paclitaxel-resistant TNBC tumor model was generated in nude mice through subcutaneous inoculation of MDA-MB-231R cells, in order to evaluate the effect of GA on the drug sensitivity and the SHH signaling pathway in vivo. As expected from the in vitro results, the combination of paclitaxel and GA significantly reduced the tumor size and inactivated the SHH signaling pathway in the xenograft tumors. In addition, the combination of paclitaxel and GA significantly enhanced the expression of cleaved caspase- 3 and BAX and reduced the expression of Bcl-2 in the xenograft tumors, compared with either treatment alone. These results were consistent with the present findings in vitro.

In conclusion, the present results revealed that GA treatment inhibited proliferation and induced apoptosis in paclitaxel-resistant TNBC cells, by inhibiting the $\mathrm{SHH}$ signaling pathway in vitro and in vivo. These findings indicated 
that GA may be a promising adjuvant drug for the therapy of paclitaxel-resistant TNBC.

\section{Acknowledgements}

Not applicable.

\section{Funding}

No funding was received.

\section{Availability of data and materials}

All data analyzed during the present study are included in this published article.

\section{Authors' contributions}

YW and YT conceived and designed the experiments. YW, YS and YT conducted all of the experiments. YW and YT wrote and revised the manuscript. All authors read and approved the final manuscript.

\section{Ethics approval and consent to participate}

All experiments involving animals were approved by the Animal Care and Welfare Committee of WeiFang People's Hospital (permit no. WF2016032702).

\section{Patient consent for publication}

Not applicable.

\section{Competing interests}

The authors declare that they have no competing interests.

\section{References}

1. Siegel RL, Miller KD and Jemal A: Cancer statistics, 2017. CA Cancer J Clin 67: 7-30, 2017.

2. Holohan C, Van Schaeybroeck S, Longley DB and Johnston PG: Cancer drug resistance: An evolving paradigm. Nat Rev Cancer 13: 714-726, 2013.

3. Zahreddine $\mathrm{H}$ and Borden KL: Mechanisms and insights into drug resistance in cancer. Front Pharmacol 4: 28, 2013.

4. Wang F, Zhang W, Guo L, Bao W, Jin N, Liu R, Liu P, Wang Y, Guo Q and Chen B: Gambogic acid suppresses hypoxia-induced hypoxia-inducible factor-1a/vascular endothelial growth factor expression via inhibiting phosphatidylinositol 3-kinase/Akt/mammalian target protein of rapamycin pathway in multiple myeloma cells. Cancer Sci 105: 1063-1070, 2014.
5. Suzuki R, Kang Y, Li X, Roife D, Zhang R and Fleming JB: Genistein potentiates the antitumor effect of 5-Fluorouracil by inducing apoptosis and autophagy in human pancreatic cancer cells. Anticancer Res 34: 4685-4692, 2014.

6. Qi Q, Lu N, Wang XT, Gu HY, Yang Y, Liu W, Li C, You QD and Guo QL: Anti-invasive effect of gambogic acid in MDA-MB-231 human breast carcinoma cells. Biochem Cell Biol 86: 386-395, 2008.

7. Li C, Qi Q, Lu N, Dai Q, Li F, Wang X, You Q and Guo Q: Gambogic acid promotes apoptosis and resistance to metastatic potential in MDA-MB-231 human breast carcinoma cells. Biochem Cell Biol 90: 718-730, 2012.

8. Li D, Song XY, Yue QX, Cui YJ, Liu M, Feng LX, Wu WY, Jiang BH, Yang M, Qu XB, et al: Proteomic and bioinformatic analyses of possible target-related proteins of gambogic acid in human breast carcinoma MDA-MB-231 cells. Chin J Nat Med 13: 41-51, 2015

9. Wang S, Wang L, Chen M and Wang Y: Gambogic acid sensitizes resistant breast cancer cells to doxorubicin through inhibiting P-glycoprotein and suppressing survivin expression. Chem Biol Interact 235: 76-84, 2015.

10. Wang X, Deng R, Lu Y, Xu Q, Yan M, Ye D and Chen W: Gambogic acid as a non-competitive inhibitor of ATP-binding cassette transporter $\mathrm{B} 1$ reverses the multidrug resistance of human epithelial cancers by promoting ATP-binding cassette transporter B1 protein degradation. Basic Clin Pharmacol Toxicol 112: 25-33, 2013.

11. Wang J and Yuan Z: Gambogic acid sensitizes ovarian cancer cells to doxorubicin through ROS-mediated apoptosis. Cell Biochem Biophys 67: 199-206, 2013.

12. Zhang W, Zhou H, Yu Y, Li J, Li H, Jiang D, Chen Z, Yang D, $\mathrm{Xu} \mathrm{Z}$ and Yu Z: Combination of gambogic acid with cisplatin enhances the antitumor effects on cisplatin-resistant lung cancer cells by downregulating MRP2 and LRP expression. Onco Targets Ther 9: 3359-3368, 2016.

13. Livak KJ and Schmittgen TD: Analysis of relative gene expression data using real-time quantitative PCR and the 2(-Delta Delta C(T)) method. Methods 25: 402-408, 2001.

14. He M, Fu Y, Yan Y, Xiao Q, Wu H, Yao W, Zhao H, Zhao L, Jiang Q, Yu Z, et al: The hedgehog signalling pathway mediates drug response of MCF-7 mammosphere cells in breast cancer patients. Clin Sci 129: 809-822, 2015.

15. Chi Y, Zhan XK, Yu H, Xie GR, Wang ZZ, Xiao W, Wang YG, Xiong FX, Hu JF, Yang L, et al: An open-labeled, randomized, multicenter phase IIa study of gambogic acid injection for advanced malignant tumors. Chin Med J (Engl) 126: 1642-1646, 2013.

16. Huang GM, Sun Y, Ge X, Wan X and Li CB: Gambogic acid induces apoptosis and inhibits colorectal tumor growth via mitochondrial pathways. World J Gastroenterol 21: 6194-6205, 2015.

17. Yang Y, Sun X, Yang Y, Yang X, Zhu H, Dai S, Chen X, Zhang H, Guo Q, Song Y, et al: Gambogic acid enhances the radiosensitivity of human esophageal cancer cells by inducing reactive oxygen species via targeting Akt/mTOR pathway. Tumour Biol 37: 1853-1862, 2016.

18. Rimkus TK, Carpenter RL, Qasem S, Chan $M$ and Lo HW: Targeting the sonic hedgehog signaling pathway: Review of smoothened and GLI inhibitors. Cancers 8: pii: E22, 2016.

19. Noble P, Vyas M, Al-Attar A, Durrant S, Scholefield J and Durrant L: High levels of cleaved caspase-3 in colorectal tumour stroma predict good survival. Br J Cancer 108: 2097-2105, 2013.

This work is licensed under a Creative Commons Attribution-NonCommercial-NoDerivatives 4.0 International (CC BY-NC-ND 4.0) License. 